\title{
Estado nutricional de la madre y la salud del niño
}

\author{
Nutritional status of the mother and the health of the child
}

\author{
Estado nutricional de la madre
}

Delia Georgina Bravo Bonoso

Universidad Estatal del Sur de Manabí, delia.bravo@unesum.edu.ec, Orcid 0000-0003-4787-8403.

Contacto: delia.bravo@unesum.edu.ec

Recibido: 25-02-2020

Aprobado: 23-06-2020

\section{Resumen}

El estado nutricional de la madre es el resultado del balance entre las necesidades y el gasto de energía alimentaria y el resultado de una gran cantidad de determinantes representado por factores; las alteraciones en la nutrición materna se reflejan frecuentemente en el retardo del crecimiento fetal, además de que existe asociación entre prematuridad con patologías maternas como: asfixia perinatal, aspiración de meconio, hipotermia, hipoglucemia, hipocalcemia y policitemia, además de las enfermedades asociadas a inmadurez y a los efectos de los factores etiológicos que produjeron el bajo peso. Este estudio fue motivado por las condiciones actuales de vida en nuestro planeta, la crisis del cambio climático, la pérdida de biodiversidad y los daños causados al agua, el aire y el suelo suscitan ahora la preocupación de si será posible alimentar de manera sostenible a esta generación de niños. Su objetivo: Describir los factores de riesgo en el estado nutricional de la madre que repercuten en la salud del niño de 0 a 5 años de edad; se empleó el método cualitativo y cuantitativo; Resultados El estado nutricional es el balance entre las necesidades y el gasto de energía alimentaria y otros nutrientes esenciales, representado por factores físicos, genéticos, biológicos, culturales, psico-socio-económicos y ambientales. Se concluye que, una ingestión insuficiente o excesiva de nutrientes, impide la utilización óptima de los alimentos ingeridos. Las causas básicas de los problemas nutricionales son: (desnutrición proteico - energética, carencias de micronutrientes y enfermedades no transmisibles relacionadas con la alimentación.

Palabras clave: incidencia, parto, embarazo alimentación, nutrición.

\section{Abstract}

The nutritional status of the mother is the result of the balance between dietary energy needs and expenditure and the result of a large number of determinants represented by factors; alterations in maternal nutrition are frequently reflected in fetal growth retardation, in addition to the fact that there is an association between prematurity and maternal pathologies such as: perinatal asphyxia, meconium aspiration, hypothermia, hypoglycemia, hypocalcemia and polycythemia, in addition to diseases associated with immaturity and to the effects of the etiological factors that produced the low weight. This study was motivated by the current living conditions on our planet, the climate change crisis, the loss of biodiversity and the damage caused to water, air and soil, now raising concerns about whether it will be possible to feed this population in a sustainable way. generation of children. Its objective: To describe the risk factors in the nutritional status of the mother that affect the health of the child from 0 to 5 years of age; the qualitative and quantitative method was used; Results The nutritional status is the balance between the needs and the expenditure of dietary energy and other essential nutrients, represented by physical, genetic, biological, cultural, psycho-socio-economic and environmental factors. It is concluded that insufficient or excessive ingestion of nutrients prevents the optimal use of the ingested food. The basic causes of nutritional problems are: (protein-energy malnutrition, micronutrient deficiencies and non-communicable diseases related to food.

Key Words: incidence, delivery, pregnancy, feeding, nutrition

\section{Introducción}

Es conocido que el estado nutricional de la gestante, antes y durante el embarazo es un factor fundamental para su salud y la de su hijo, situación importante a ser considerada, una vez que estas mujeres constituyen un grupo nutricionalmente vulnerable.

Según datos de la Encuesta Nacional de Salud y Nutrición (ENSANUT), el 28,8\% de las mujeres adolescentes presentan sobrepeso y obesidad. El promedio de talla de la mujer ecuatoriana es de 151,2 $\mathrm{cm}$; el $13,8 \%$ tiene menos de 145 centímetros, considerado como baja talla. Las madres pequeñas tienen mayores probabilidades de tener niños pequeños. 


\section{HIGIA DE LA SALUD}

La anemia en la mujer embarazada sigue presente en nuestro país. De acuerdo a estudios de puestos centinela del MSP, el 46,9\% de mujeres embarazadas presenta anemia, lo cual ocasiona problemas en las mujeres y en el recién nacido: como bajo peso al nacer e incremento de la mortalidad perinatal.(1)

La nutrición materna antes del embarazo es reconocida como un factor importante del resultado del nacimiento. Existe una relación directa entre la masa corporal de la madre y el desarrollo de la masa corporal del producto. Mujeres con bajo peso para su talla, presentan una serie de peligros para su producto, entre los que se cuentan la desnutrición fetal y el bajo peso al nacer, lo que aumenta varias veces el riesgo de morbilidad neonatal e infantil, causa trastornos familiares y sobrecarga los presupuestos de los servicios de cuidados intensivos y neonatales. Estos efectos se incrementan si además, la gestante experimenta poca ganancia de peso durante el embarazo y muestra bajos valores hemoglóbicos.(2)

El estado nutricional es, primariamente, el resultado del balance entre las necesidades y el gasto de energía alimentaria y otros nutrientes esenciales, y secundariamente, el resultado de una gran cantidad de determinantes en un espacio dado representado por factores físicos, genéticos, biológicos, culturales, psico-socio-económicos y ambientales. Estos factores pueden dar lugar a una ingestión insuficiente o excesiva de nutrientes, o impedir la utilización óptima de los alimentos ingeridos. (3)

Son tres las causas básicas de los problemas nutricionales (desnutrición proteico - energética, carencias de micronutrientes y enfermedades no transmisibles relacionadas con la alimentación).

Alimentos: disponibilidad, acceso y consumo de alimentos inocuos y de buena calidad;

Salud (utilización biológica): estado personal de salud, estilo de vida y utilización de los servicios de salud;

Cuidados: capacidad de la familia y de la comunidad para cuidar de las personas vulnerables o dedicar tiempo, atención, ayuda y conocimientos prácticos para cubrir las necesidades de estas personas.

Nutrición: es un proceso de extraordinaria complejidad biológica que se refiere a la distribución, utilización, transformación, almacenamiento y/o eliminación de los nutrimentos en el organismo.

Dieta: no es más que el conjunto de categorías químicas, alimentarias y nutrimentales, que puede ser ingerida por el individuo con el propósito de satisfacer sus necesidades energéticas y sustanciales.

El periodo de gestación es un proceso fisiológico normal que permite la supervivencia de la especie y a su vez presenta también una trascendencia nutricional, porque para la formación del nuevo ser, es preciso un aporte de alimentos que deben ser adecuados en cantidad, calidad y suministrados a lo largo del embarazo para que el niño nazca con peso normal. En Ecuador, 1 de cada 4 niños y niñas menores de 5 años sufre desnutrición crónica. La situación es más grave
Periodo. Enero - Junio 2020

Vol. 2, Nro. 1, Publicado 2020-06-30

para la niñez indígena: 1 de cada 2 niños la padece y 4 de cada 10 presentan anemia.

La oportuna valoración nutricional de la gestante juega un papel importante para el diagnóstico precoz, prevención, promoción y tratamiento de riesgos como: la anemia, bajo peso al nacer, macrosomía fetal a causa del inadecuado estado nutricional de las embarazadas. La educación y asesoramiento nutricional es una estrategia que se utiliza con mucha frecuencia para mejorar el estado nutricional de las mujeres durante el embarazo.

El desafío de los profesionales de la salud es contribuir a establecer prácticas de vida saludable con relación a la alimentación, garantizando que se cumplan los requerimientos nutricionales de la madre y del niño. Frente a esta realidad y por tratarse de un tema importante para la sociedad en general, este trabajo investigativo procuró describir los factores de riesgo en el estado nutricional de la madre que repercuten en la salud del niño de 0 a 5 años de edad; la cual se basa en un estudio de caso de un infante de sexo femenino, con antecedentes maternos de problemas nutricionales durante el embarazo, con la finalidad de analizar las repercusiones que origina el estado nutricional de la madre en la salud del niño.

\section{Materiales y métodos.}

La investigación es de tipo cualitativa es una técnica descriptiva de recopilación de datos que se utiliza para descubrir detalles que ayudan a explicar el comportamiento. Los datos cualitativos son todo lo que se describe o explica, desde observaciones de una interacción hasta citas de personas sobre sus experiencias, actitudes, creencias y pensamientos, las técnicas utilizadas fueron; la observación, entrevista clínica, método de exploración o examen físico, método de revisión de la historia o expediente clínico y sesiones de grupo.

La observación tiene por objetivo examinar los factores externos que puedan afectar al paciente, a su vez influye en la realización del examen físico, el mismo que ayuda a obtener información más concisa. La entrevista ayuda a responder incógnitas a través de preguntas planteadas, es un método que apoya en la recolección de datos personales del paciente en la elaboración de la anamnesis. La Muestra constituida por 1 caso actual.

Como criterios de inclusión se consideraron las revisiones de casos clínicos, a fines a la investigación y con resultados de impactos y se consideró a 1 paciente que quisiera ser partícipe del estudio, como criterios de exclusión revisiones bibliográficas con resultados de poco impacto.

Consideraciones éticas, previo a la información respectiva, acerca de las distintas implicaciones del estudio, se solicitó consentimiento informado, mismo que incluía la información necesaria para que las madres decidan participar o no en el estudio y responder la encuesta y para la toma de los indicadores antropométricos.

\section{Descripción del caso clínico}




\section{HIGÍA DE LA SALUD}

Infante de 2 años de edad, sexo femenino, acude al centro de salud acompañado de su madre, la madre refiere que presenta diarrea, vómito y fiebre, llanto y cansancio hace poco de una semana. Se observa fascias pálidas y poco hidratadas, labios resecos y decaimiento. Signos vitales: temperatura; $38^{\circ} \mathrm{C}$, frecuencia cardiaca; 95 por minuto, frecuencia respiratoria 40 por minuto,
Periodo. Enero - Junio 2020

Vol. 2, Nro. 1, Publicado 2020-06-30 presión arterial; 70/ $50 \mathrm{MmHg}$, peso; 7,1 kg y talla; 0.70 $\mathrm{cm}$. Antecedentes de importancia: Madre de infante menciona haber tenido problemas nutricionales durante el embarazo. A partir de los 6 meses de edad el paciente empezó a consumir alimentos básicos; arroz, sopitas, coladas, jugos y lo que estaba al alcance de ella por la carencia de recursos económicos.

Tabla. 1 Anamnesis del infante

\begin{tabular}{|l|l|}
\hline \multicolumn{2}{|l|}{ Datos generales } \\
\hline Datos de identificación: & Isabella Smith \\
\hline Nombre y apellidos: & $131724 * * * * *$ \\
\hline Numero de cedula: & Ecuatoriana \\
\hline Domicilio: & Manta-Manabí-Ecuador \\
\hline Estado civil: & ------ \\
\hline Edad: & 2 años \\
\hline Tipo de sangre: & O RH + \\
\hline Fecha de nacimiento & 02 de agosto de 2018 \\
\hline Sexo: & femenino \\
\hline Ocupación: & --------- \\
\hline Religión: & ----------- \\
\hline Perfil del paciente & \\
\hline
\end{tabular}

Paciente de sexo femenino con 2 años de edad residente de la ciudad de Manta. Vive con abuelos y madre.

\section{Motivo de la consulta}

Presenta diarrea, vómito y fiebre, llanto y cansancio hace poco de una semana

\section{Historia de la enfermedad actual}

Paciente de sexo femenino de 2 años de edad, con antecedentes de desnutrición aguda, acompañada de cansancio, diarrea, vómito, fiebre y perdida de $2 \mathrm{~kg} 1$ mes.

\section{Perfil de infante:}

Composición familiar: actualmente vive con abuelos y madre.

Mamá

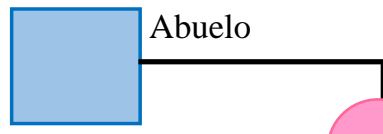

Abuela

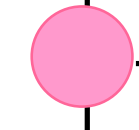

paciente de 2 años

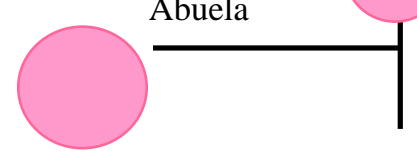

Estilo de vida

Infante de 2 años de edad, despierta a las 8:00 AM, toma biberón y recibe aseo por parte de la madre, luego juega y consume frutas siempre que se pueda. A la hora del almuerzo toma sopita de pollo, cremas o de queso, se entretiene con la televisión y sus juguetes mientras la madre realiza las tareas del hogar. Se le realiza aseo 
personal. Interactúa un rato con la madre. A las 6:00 PM se le cambia de ropa y se realiza aseo, también consume su cena papilla y después de una hora su leche de formula. Descansa a las 9:00 PM.

- Alimentación:

Lactancia mediante formula y alimentación complementaria.

- Socioeconómico y epidemiológicos:

Habita en casa de sus abuelos que posee estructura de madera, piso con cemento, con mala ventilación, buena iluminación, cuenta con una sala pequeña, comedor, 1 baño, 2 dormitorios y una cocina. Tiene poca condición de higiene, cuenta con servicio de agua con tratamiento adecuado y luz eléctrica.

\section{Antecedentes patológicos personales}

Producto de la concepción a término bajo peso al nacer.

\begin{tabular}{|l|l|}
\hline Inmunización: & Esquema de vacunas completas para la edad \\
\hline Sintomatología: & Cansancio, diarrea, vómito, fiebre y llanto \\
\hline
\end{tabular}

\section{Antecedentes patológicos familiares}

Madre: Anemia severa durante el embarazo

Abuela: Hipertensión arterial

Abuelo: Diabetes mellitus

Diagnóstico; Desnutrición aguda

\section{EXAMEN FÍSICO}

Signos vitales:

- $\quad$ T/A: $70 / 50 \mathrm{mmHg}$

- FR: 40x

- FC: $95 x^{\wedge}$

- $\mathrm{T}^{\mathrm{o}}: 38^{\circ} \mathrm{C}$

\section{Antropometría}

- Peso: $7,1 \mathrm{~kg}$

- Talla: $0.70 \mathrm{~cm}$

- IMC: 14.4

- Perímetro cefálico: $44 \mathrm{~cm}$

- Perímetro torácico: $40.5 \mathrm{~cm}$

- Circunferencia del brazo: $12 \mathrm{~cm}$

Aspecto general: Lactante longilínea asténico fascias pálidas y poco hidratadas, labios resecos ausencia de cicatrices, no presenta eritemas, con buena textura y elasticidad, cabello con buena higiene de color negro con poca implantación, reseco y quebradizo.

\section{Cabeza}

Cráneo: Simétrico normolíneo con buena implantación de cuero cabelludo, fontanelas bregmáticas de forma cuadrangular sin alteraciones ni deformaciones, ausencia de pediculosis, ausencia de dolor al momento de la palpación se logra parpar las arterias temporales, perímetro cefálico de $44 \mathrm{~cm}$.

Cara: Simétrica de acuerdo a la estatura y peso corporal del lactante, facies pálidas y expresivas, ausencia de lesiones, piel reseca sin movimientos involuntarios ni edemas, temperatura corporal de $38^{\circ}$ $\mathrm{C}$, ausencia de dolor al momento de la palpación.

Ojos: Ojos hundidos, parpadeo bilateral reflejo corneal positivo, parpados simétricos, pestañeo espontaneo pupilas isocóricas, esclerótica de coloración blanca, iris de color negra, cejas simétricas, sacos lacrimales no inflamados.
Oídos: Orejas simétrica de forma alargada, pabellón auricular con buena implantación, ausencia de masas reacciona ante sonidos emitidos por parte del examinador.

Nariz y senos paranasales: nariz simétrica, vías aéreas poco permeables, ausencia de secreciones no presenta masas ni protuberancias, ausencia de aleteo nasal no presenta dolor al momento de la palpación frecuencia respiratoria de $40 \mathrm{x}$

Boca: Simétrica, ausencia de masas y lesiones, labios resecos y coloración pálida ausencia de buena integridad, mucosas deshidratadas, ausencia de masas encías no inflamadas sin presencia de alguna pieza dentaria hasta el momento.

Cuello: cuello asimétrico móvil, corto de volumen y posición central en relación con el resto del cuerpo, no se encuentra acorde al biotipo y edad del infante, ausencia de masas no presenta tumoraciones, pulsos carotideos palpables de buena forma e intensidad, ausencia de adenopatías.

Tórax y pulmones: Caja torácica simétrica no presenta masas ausencia de dolor al momento de la palpación, ausencia de obstrucción pulmonar frecuencia respiratoria $40 x^{`}$

Cardiovascular: Ausencia de taquicardia, presión arterial estable, ausencia de edemas, T/A 70/50 MmHg. Abdomen: Depresible al momento de la palpación, ausencia de hernias, presencia de peristaltismo, doloroso a la palpación.

Gastrointestinal: Deposiciones de color amarillo verdoso con olores fétidos, presenta náuseas y vómitos. Presenta dolores en la región abdominal.

Genitales femeninos: de acuerdo a su edad sin alteraciones.

Músculos esqueléticos: extremidades inferiores y superiores simétricas, piel normo colorida ausencia de lesiones sin antecedentes personales de fracturas 
ISSN 2773-7705

\section{HIGIA DE LA SALUD}

Neurológicos: Lactante orientado, responde al test de Denver sin ninguna complicación.

Análisis de la aplicación del Test de Denver

Con la aplicación del Test de Denver se valoró cuatro áreas del desarrollo psicomotor del niño las cuales son; el desarrollo personal-social, motor fino-adaptativo, lenguaje y motor grueso. Con la aplicación de este test ya estructurado se puede evidenciar que la niña responde en un $85 \%$ a su capacidad y motricidad que corresponden a los 2 años de vida. El examen físico e interacción de objetos ayudaron a examinar las habilidades motoras de la niña, demostrando un moderado desarrollo en sus habilidades y destrezas desde lo más simple a lo más complejo, con movimientos musculo-esqueléticos coordinados según su capacidad psíquica acorde a su edad.

Estudios de apoyo diagnóstico y resultados

- Primero se realiza la evaluación médica. Esta se realiza, mediante la formulación de preguntas acerca de la alimentación, selección y forma de preparación, pérdida de peso y un examen físico. Las respuestas pueden ayudar a confirmar el diagnóstico y a identificar la causa. Mediante el examen físico se realiza la toma de medidas antropométricas y otros síntomas como cambios en la piel y el cabello.

- Dependiendo de la evaluación médica se realiza análisis de sangre, para medir el nivel de albúmina (rango normal es de 3.4 a 5.4 $\mathrm{g} / \mathrm{dL}$ ) (que disminuye cuando una persona no consume suficiente proteína). También se puede medir el número de determinados tipos de glóbulos blancos (que disminuye a medida Tratamiento que la desnutrición empeora).

Tabla 2 Plan de Cuidados según Nanda-NIC-NOC basado en el modelo de atención de Virginia Henderson

\begin{tabular}{|c|c|c|c|c|}
\hline Valoración: & \multicolumn{4}{|c|}{$\begin{array}{l}\text { Gordon } \\
\text { Dominio: } 0002 \text { NUTRICIÓN } \\
\text { Clase: } 0001 \text { INGESTIÓN }\end{array}$} \\
\hline Valoración: & \multicolumn{4}{|c|}{$\begin{array}{l}\text { Henderson } \\
\text { Necesidad: II ALIMENTACION / HIDRATACION }\end{array}$} \\
\hline Cód. Diagnóstico: & \multicolumn{4}{|l|}{00002} \\
\hline Diagnóstico: & \multicolumn{4}{|c|}{ Desequilibrio Nutricional por defecto } \\
\hline Definición: & \multicolumn{4}{|c|}{ Ingesta de nutrientes insuficiente para satisfacer las necesidades metabólicas. } \\
\hline $\begin{array}{c}\text { Diagnostico } \\
\text { NANDA }\end{array}$ & $\begin{array}{c}\text { NOC } \\
\text { Resultados }\end{array}$ & $\begin{array}{c}\text { NIC } \\
\text { Intervenciones }\end{array}$ & $\begin{array}{c}\text { Razonamiento } \\
\text { científico }\end{array}$ & Evaluación \\
\hline $\begin{array}{c}00002 \\
\text { Desequilibrio } \\
\text { Nutricional } \\
\mathbf{R} / \mathbf{C} \\
\text { Incapacidad para } \\
\text { absorber los nutrientes } \\
\mathbf{M} / \mathbf{P} \\
\text { Pérdida de peso } \\
\text { con un aporte } \\
\text { nutricional adecuado }\end{array}$ & $\begin{array}{l}1009 \text { Estado } \\
\text { nutricional } \\
\text { ingestión de } \\
\text { nutrientes }\end{array}$ & $\begin{array}{c}1100 \text { Manejo de la } \\
\text { nutrición } \\
1240 \text { Ayuda para } \\
\text { ganar peso } \\
5246 \text { Asesoramiento } \\
\text { nutricional }\end{array}$ & $\begin{array}{l}\text { - Ayuda a proporcionar una } \\
\text { dieta equilibrada de sólidos } \\
\text { y liquidos. } \\
\text { - Facilitar el aumento de } \\
\text { peso corporal. } \\
\text { - Utilización de un proceso } \\
\text { de ayuda interactivo } \\
\text { centrado la necesidad de } \\
\text { modificación de la dieta. }\end{array}$ & $\begin{array}{l}\text { - La madre refiere } \\
\text { que ha recibido } \\
\text { información } \\
\text { suficiente acerca } \\
\text { de cómo mejorar } \\
\text { la alimentación y } \\
\text { relata haber tenido } \\
\text { resultados } \\
\text { favorables. }\end{array}$ \\
\hline
\end{tabular}

\section{Evolución}

Recibo paciente de sexo femenino de 2 años de edad, acompañada de su madre, con antecedentes de desnutrición aguda, consciente, orientada en tiempo espacio y persona, acompañada de cansancio, disentería, emesis, fiebre la madre refiere que la niña tiene pérdida de peso. Con signos vitales T/A: 70/50 MmHg, FR: 40x`, FC: $95 x^{\prime}, \mathrm{T}^{\circ}: 38^{\circ} \mathrm{C}$, se le administra cloruro de potasio, amoxicilina, Gentamicina. Se realiza tramite de alta, se le brinda orientación sobre los cuidados y la nutrición a seguir en el hogar, se retira en silla de ruedas consciente orientada en tiempo espacio y persona, acompañada de su madre.

- Administrar en 4 a 6 horas: $75 \mathrm{ml} / \mathrm{kg}$ de SRO de baja osmolaridad sin modificaciones. continuas: $10 \mathrm{ml} / \mathrm{kg} / \mathrm{hr}$ de SRO de baja potasio a un litro de esta SRO.

90 mg/kg/día VO o SNG MÁS Gentamicina 5 kg/día IM o IV

- Vigilar gasto urinario, estado de consciencia, Continuar la reposición de las pérdidas a razón de 50-100 $\mathrm{ml}$ de suero de rehidratación por cada deposición líquida.

minutos durante las primeras dos horas y luego cada hora.

- Signos de sobre hidratación son: aumento de la frecuencia cardiaca, aparición de ronquido respiratorio, hepatomegalia e ingurgitación venosa. 


\section{HIGIA DE LA SALUD}

Análisis: De acuerdo al estudio de caso se manifiesta, que las principales causas de desnutrición son la ingesta inadecuada de nutrientes, las condiciones socioecomicas del hogar y el tipo de calidad de vida. Encontrando un efecto negativo de la lactancia materna, además es importante tener en cuenta que los niños con un estado nutricional normal tienen un promedio de nueve meses de lactancia materna y aquellos con un promedio mayor sufren de desnutrición crónica La mala alimentación de la madre durante el embarazo debido a su pobreza está afectada la calidad de vida del niño y esto se ve demostrado en sus medidas antropométricas en las cuales se determinó que la paciente tiene un bajo peso. Se puede determinar que existe una relación tanto entre el estado socioeconómico y desarrollo psicomotor. También un factor determinante que podría influenciar al desarrollo
Periodo. Enero - Junio 2020

Vol. 2, Nro. 1, Publicado 2020-06-30

psicomotor es la educación de los padres que se ve ligada al nivel socioeconómico.

\section{Conclusiones}

Pudimos cumplir con nuestro objetivo profesional de poder contribuir en la recuperación de salud de una persona ya que elaboramos actividades para poder llegar a esa meta, en este caso hablamos de la desnutrición infantil y así poder conocer más de la enfermedad del paciente y ayudarlo a su pronta recuperación.

Se puede concluir también que la pobreza y la desnutrición son dos problemas centrales que afectan directamente a nuestra paciente, esta problemática tiene repercusiones graves, por causa de que las personas a las que afecta generalmente y en mayor rango es a niños, produciendo graves martirios, bajo rendimiento escolar y es un a emergencia invisible por el peligro mortal que está implicado.

\section{Colaboradores de la Investigación:}

Gina Stefania Alay Soledispa, María José Ayón Victores, Yuletsi Estefanía Bozada Cedeño, Yordy Alexander Bozada Cox, Valeria Cecibel Cabrera Carreño, Yilari Takeshy Cantos Chavarría, Flerida Annabel Merchán Pibaque, Evelyn Alexandra Muñiz Villafuerte, Denisse Mariby Pillasagua López, Yelena Dolores Piloco Pincay.

\section{Bibliografía}

1. Ghebreyesus DTA. Organización Mundial de la Salud. [Online].; 2020 [cited 202008 18. Available from: https://www.who.int/topics/nutrition/es/.

2. Pedraza DF. Estado nutricional como factor y resultado de la seguridad alimentaria y nutricional y sus representaciones en Brasil. Scielo-Salud Pública. 2015 Junio; 1(231).

3. Sidhu S. UNICEF. [Online].; 2019 [cited 202008 18. Available from: https://www.unicef.org/es/comunicadosprensa/la-mala-alimentacion-perjudica-la-salud-de-los-ninos-en-todo-el-mundo.

4. Noemi MMJ. Estado nutricional y prácticas alimentarias durante el embarazo en las gestantes a término atendidas en el Instituto Nacional Materno Perinatal. informar. Lima-Perú: UNIVERSIDAD NACIONAL MAYOR DE SAN MARCOS, FACULTAD DE MEDICINA-E.A.P. DE OBSTETRICIA; 2016. Report No.: 1.

5. Pública MdS. MINISTERIO DE SALUD PÚBLICA. [Online]; 2014 [cited 202008 19. Available from: file://C:/Users/Personal/Downloads/Alimentacion_y_nutricion_de_la_mujer_gestante_y_la_madre_en_period o_de_lactancia(1).pdf.

6. UNICEF. UNICEF. [Online].; 2019 [cited 202008 19. Available from: https://www.unicef.org/es/comunicadosprensa/la-mala-alimentacion-perjudica-la-salud-de-los-ninos-en-todo-el-mundo.

7. Redacción comercial. Primicias. [Online].; 2020 [cited $2020 \quad 08 \quad 19$. Available from: https://www.primicias.ec/noticias/patrocinado/embarazo-y-mal-nutricion-no-se-llevan-bien/.

8. REVILLA-C.BLACK-J.HOLMES G. OMS. [Online].; 2017 [cited 202008 19. Available from: https://www.who.int/features/factfiles/nutrition/es/.

9. HEREDIA V. LA DESNUTRICIÓN CRÓNICA EN NIÑOS AUMENTÓ EN ECUADOR. EL SOBREPESO Y LA OBESIDAD TAMBIÉN SE INCREMENTÓ. 2020 Jan 07: p. 2.

10. Morley JE. MANUAL MSD. [Online]; 2018 [cited $2020 \quad 08$ 19. Available from: https://www.msdmanuals.com/es-ec/hogar/trastornos-nutricionales/desnutrici\%C3\%B3n/desnutrici\%C3\%B3n. 